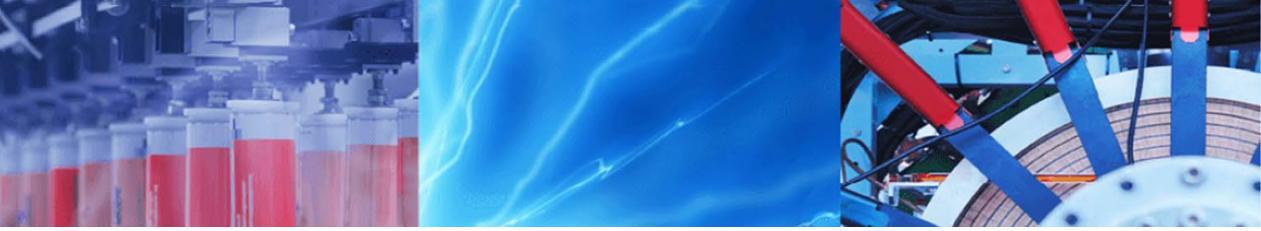

Research Article

\title{
Designing of nickel cobalt molybdate/multiwalled carbon nanotube composites for suppression of electromagnetic radiation
}

\author{
Preetam Bhardwaj $^{1} \cdot$ Shivani Kaushik ${ }^{1} \cdot$ Preeti Gairola $^{1,2} \cdot$ S. P. Gairola ${ }^{1}$
}

(c) Springer Nature Switzerland AG 2018

\begin{abstract}
One-dimensional nanorod structures of cobalt molybdate, nickel cobalt molybdate and nickel cobalt molybdate/multiwalled carbon nanotube composites have been effectively synthesized by mild hydrothermal method. The elemental analysis of prepared composite nanorods is studied by Fourier transform infrared and electron diffraction X-ray spectroscopy. Structures and morphologies of samples are characterized by X-ray diffraction and field emission scanning electron microscope. SEM morphology authenticates the development of nanorod structures of synthesized samples. The particles' size attained by $\mathrm{CoMoO}_{4}, \mathrm{NiCoMoO}_{4}$ and $\mathrm{NiCOMoO}_{4} / \mathrm{CNTs}$ from SEM morphology is in the range of 45-65 nm, 75-85 nm and $100-125 \mathrm{~nm}$. It is experiential that after Ni doping in $\mathrm{CoMoO}_{4}$ particle size is increased and constituent parts are transformed into consistent dimension with better morphology. The SEM image of $\mathrm{NiCoMoO}_{4} / \mathrm{CNTs}$ shows the threadlike structures that reveal the presence of carbon nanotubes in the composite. EMI shielding measurements were taken using pressed rectangular pellets of $2 \mathrm{~mm}$ thickness. The measured total electromagnetic interference (EMI) shielding efficiency $\left(\sim \mathrm{SE}_{\mathrm{T}}\right)$ of $\mathrm{NiCoMoO}_{4} / \mathrm{CNTs}$ is $48.84 \mathrm{~dB}$ in the Ku band $(12.4-18.0 \mathrm{GHz})$, and involvement of SE due to the absorption of total EMI SE of the composite is larger than that of reflection. This is due to the development of strong conducting network through MWCNTs within divalent transition metal-doped metal-metal matrix. The obtained results suggest the usefulness of the composite for commercial use in far-field EMI shielding and suppression of electromagnetic radiation.
\end{abstract}

Keywords Cobalt molybdate $\cdot$ Multiwalled carbon nanotubes $\cdot$ Electromagnetic radiation $\cdot$ Hydrothermal method

\section{Introduction}

Nowadays, existing technologies have become a source of wide-ranging electromagnetic (EM) smog originated from electromagnetic fields and electromagnetic radiations. In many cases, this electromagnetic smog is much stronger than any natural sources of electromagnetic fields or radiations. Currently, electromagnetic smog has been aggravating at a recognizable rate due to the expansion of various electronics devices, such as cellular phones, tablet PCs and wireless local area networks. EM smog may cause operational collapse within the electronic neighbourhood and shatter the intrinsic EM field of human beings. The associated hazards may be a loss of money, energy sources, time or even precious human life. The mischief caused by this electromagnetic smog has pessimistic influence on human beings [1-3]. Therefore, highperformance electromagnetic interference (EMI) shielding materials are required to sort out the internal electronics from the vicinity. Theoretically, when the incident EM radiations drive into shielding materials, they will be impaired by three dissipation ways: reflection loss, absorption loss and internal multiple reflection loss. A device is advised electromagnetically compatible with its vicinity if it does not interfere with other devices or itself, and it does not feigned by emissions from other devices. Therefore, a good shielding material require for restrain both incoming and outgoing electromagnetic interferences (EMIs). In recent years, low-dimensional shape-controlled nanocrystals have been a passionate

$\triangle$ S.P. Gairola, spgairola10@gmail.com | 'Research and Development Cell, Uttaranchal University, Prem Nagar, Dehradun, India. ${ }^{2}$ Department of Physics, Gurukula Kangri Vishwavidyalaya, Haridwar, India.

SN Applied Sciences (2019) 1:113 | https://doi.org/10.1007/s42452-018-0115-7

Received: 15 October 2018 / Accepted: 7 December 2018 / Published online: 18 December 2018 
centre of attention of the research in nanotechnology for their exclusive physical and chemical properties, and their probable technological applications $[4,5]$. The synthesized nanomaterials have been a significant and sizzling research topic in the high-tech applications because of their elevated surface area-to-volume ratio, fewer agglomerated configuration, abridged diffusion paths and fast redox reactions in the solid phase [6-8]. One-dimensional nanorod nanostructures are perfect structure blocks for nanoscale electronics, optoelectronics and sensing devices; meanwhile, they signify huge surface area, small space for mass and charge transport, and independence for volume change [9-12]. Metal molybdate, $\mathrm{AMoO}_{4}$-type compounds (where $\mathrm{A}$ is a divalent metal ion), is an attention-grabbing research subject owing to their possible application in many fields, such as magnetic properties, photoluminescence and catalysts [13-17]. Furthermore, the discovery of carbon nanotubes (CNTs) with the outstanding mechanical, electrical and thermal properties prepared them as a prospective applicant for high-tech applications. Being deprived dispersal and being deficient in interfacial linkage in the nature of CNTs possess severe impediments to their further development. To resolve the above problems, functionalization routes have been developed [18] that often apparent themselves in some worsening of intrinsic properties of pristine CNTs [19]. An immense attention is been given to CNTs as filler in EMI shielding composite material due to its distinctive aperture structure, high-quality mechanical and thermal stability and advanced electrical properties [20].

In this work, we have successfully synthesized cobalt molybdate, nickel cobalt molybdate and nickel cobalt molybdate/multiwalled carbon nanotube composites by interfacial hydrothermal method. The electromagnetic radiations suppression properties of synthesized nanorod composites were studied systematically by vector network analyser method. We also investigated effect of nickel doping on cobalt molybdate and CNT incorporation within metal-metal molybdate matrix that has a remarkable effect on the EMI shielding behaviour of the samples. The EMI shielding measurements showed that prepared samples exhibit good shielding effectiveness characteristics in $\mathrm{Ku}$ band. This enhanced EMI shielding performance of nanorods might be attributed to their high conductivity, enlarged surface area and well-organized electron transport path, which were facilitated by one-dimensional nanostructure.

\section{Experimental work}

\subsection{Material}

Cobalt nitrate $\left(\mathrm{Co}\left(\mathrm{NO}_{3}\right)_{2} \cdot 6 \mathrm{H}_{2} \mathrm{O}\right)$, nickel nitrate $\left(\mathrm{Ni}\left(\mathrm{NO}_{3}\right)_{2} \cdot 6 \mathrm{H}_{2} \mathrm{O}\right)$, ammonium molybdenum $\left(\left(\mathrm{NH}_{4}\right)_{6} \mathrm{Mo}_{7} \mathrm{O}_{24} \cdot 4 \mathrm{H}_{2} \mathrm{O}\right)$ and multiwalled carbon nanotubes (MWCNTs) were bought from
Sigma-Aldrich, Germany. Other chemical compounds, like nitric acid, methanol, sulphuric acid, were utilized as received, and all were of analytical grade. Aqueous solutions were prepared using deionized water.

\subsection{Synthesis of nanorod ternary composites}

\subsubsection{Scheme 1: Synthesis of $\mathrm{CoMoO}_{4} /$ composite}

The cobalt molybdate $\left(\mathrm{CoMoO}_{4}\right)$ composite is synthesized by hydrothermal method. The synthesis protocol was as follows: $0.04 \mathrm{M}$ of ammonium molybdenum solution was suspended in $50 \mathrm{ml}$ deionized water, and similarly, $0.04 \mathrm{M}$ of cobalt nitrate was dissolved in $50 \mathrm{ml}$ deionized water. The cobalt nitrate solution was added into the ammonium molybdenum solution and magnetic stirred for $12 \mathrm{~h}$ which fabricates a homogenous solution of $\mathrm{pH} 6$. The reaction mixture was then transferred to a Teflon-lined stainless autoclave. The autoclave was maintained at $160^{\circ} \mathrm{C}$ for $24 \mathrm{~h}$ in an oven and then cooled naturally to room temperature. The dark purple-coloured precipitate was collected, filtered and washed with deionized water and ethanol several times. Then the sample was dried in oven at $60^{\circ} \mathrm{C}$ for $6 \mathrm{~h}$.

\subsubsection{Scheme 2: Synthesis of $\mathrm{NiCoMoO}_{4} /$ composite}

The nickel cobalt molybdate $(\mathrm{NiCoMoO})_{4}$ composite was synthesized by same hydrothermal method. Here $0.04 \mathrm{M}$ of ammonium molybdenum solution was dissolved in $50 \mathrm{ml}$ deionized water and equal moles $(0.04 \mathrm{M})$ of cobalt nitrate and nickel nitrate were prepared each in $50 \mathrm{ml}$ deionized water. The cobalt nitrate and nickel nitrate solutions were added into the ammonium molybdenum solution and magnetic stirred for $12 \mathrm{~h}$. The mixture was transferred to a Teflonlined stainless autoclave. The autoclave was maintained at $160^{\circ} \mathrm{C}$ for $24 \mathrm{~h}$. The light purple-coloured precipitate was obtained, filtered and washed with deionized water and ethanol several times. After drying in an oven at $60^{\circ} \mathrm{C}$ for $6 \mathrm{~h}$, the final product was obtained.

\subsubsection{Scheme 3: Synthesis of $\mathrm{NiCoMoO}_{4} / \mathrm{CNT}$ composite}

The similar above-said hydrothermal synthesis reaction was adopted to synthesize nickel cobalt molybdate/CNT composite by two-step processes. In the first step, MWCNTs were functionalized by oxidation in a mixture of $\mathrm{H}_{2} \mathrm{SO}_{4}$ and $\mathrm{HNO}_{3}$ (1:3 volume ratio) for $4 \mathrm{~h}$ with ultrasonication. The acid-treated MWCNTs were vacuum-filtered and washed with deionized water and absolute ethanol 3-5 times and then dried in an oven at $60^{\circ} \mathrm{C}$ for $6 \mathrm{~h}$.

Thus, acid-treated MWCNTs were obtained. In the second step, $0.04 \mathrm{M}$ ammonium molybdenum was dissolved in $50 \mathrm{ml}$ of deionized water. Equal moles $(0.04 \mathrm{M})$ of 
cobalt nitrate and nickel nitrate solution were prepared each in $50 \mathrm{ml}$ of deionized water. The cobalt nitrate solution was added into the ammonium molybdenum solution and magnetic stirred for $12 \mathrm{~h}$. Then $0.1 \mathrm{~g}$ of CNTs was added in the prepared solution and the mixture was ultrasonicated for $1 \mathrm{~h}$. Then the reaction mixture was transferred to a Teflon-lined stainless autoclave. The hydrothermal reaction was kept at $160^{\circ} \mathrm{C}$ for $24 \mathrm{~h}$, and after that, autoclave was cooled down to room temperature. Then precipitate was filtered and repeatedly washed with deionized water for 3-4 times. Finally, grey-coloured precipitate was obtained and then dried in an oven at $60{ }^{\circ} \mathrm{C}$ for $12 \mathrm{~h}$. Figure 1 shows schematic illustration of preparation of cobalt molybdate, nickel cobalt molybdate and nickel cobalt molybdate/carbon nanotube composites and shielding effectiveness measurement by vector network analyser system.

\subsection{Characterization}

X-ray powder diffraction patterns (XRD) of the composite samples were traced by a D8 diffractometer in the $2 \theta$ range of $20^{\circ}-70^{\circ}$ by using a Japan Rigaku Ultima III $\mathrm{X}$-ray diffractometer with graphite monochromatized $\mathrm{Cu}$ Ka radiation $(\lambda=1.5418 \AA)$. The field emission scanning electron microscope (FE-SEM) measurements were taken with a field emission microscope (JEOL 6701F equipped with a Bruker EDX detector) at an acceleration voltage of $5.0 \mathrm{kV}$. The EMI shielding measurements were taken using pressed rectangular pellets ( $2 \mathrm{~mm}$ thick) placed inside the homemade sample holder. The holder matches the internal dimensions of $\mathrm{Ku}$ band $(12.4-18.0 \mathrm{GHz})$ waveguide placed between the two ports of vector network analyser (VNA E8263B, Agilent Technologies).

\section{Results and discussions}

\subsection{Physicochemical characteristics of cobalt molybdate, nickel cobalt molybdate and nickel cobalt molybdate/CNT composite}

Fourier transform infrared (FTIR) spectroscopy studies of the cobalt molybdate, nickel cobalt molybdate and nickel cobalt molybdate/CNT composite are completed using a FTIR spectrophotometer between the range of 400 and $4000 \mathrm{~cm}^{-1}$. FTIR was used to further examine the phase transparency and structural bonding related to the functional groups (Fig. 2). The FTIR spectra are shown in Fig. 2. The broad absorption band in the region around 3418 and $3425 \mathrm{~cm}^{-1}$ is owing to the existence of coordinated/ entrapped water. The occurrence of some carbonaceous material is obvious from the IR spectra which illustrate strong band at 1595 and $1364 \mathrm{~cm}^{-1}$ consequent to carboxylate ions. The band around 775 and $777 \mathrm{~cm}^{-1}$ corresponds to being modes of vibration of cobalt and that around 647 and $644 \mathrm{~cm}^{-1}$ corresponds to bending modes of vibration of nickel $[4,5]$. The other bands may be due to the microstructure formation of the sample. For the $\mathrm{a}-\mathrm{MoO}_{4}$ material, well-known absorption bands at 1120 ,
Fig. 1 Schematic illustration of preparation of cobalt molybdate, nickel cobalt molybdate and nickel cobalt molybdate/ carbon nanotube composites and shielding effectiveness measurement by vector network analyser system

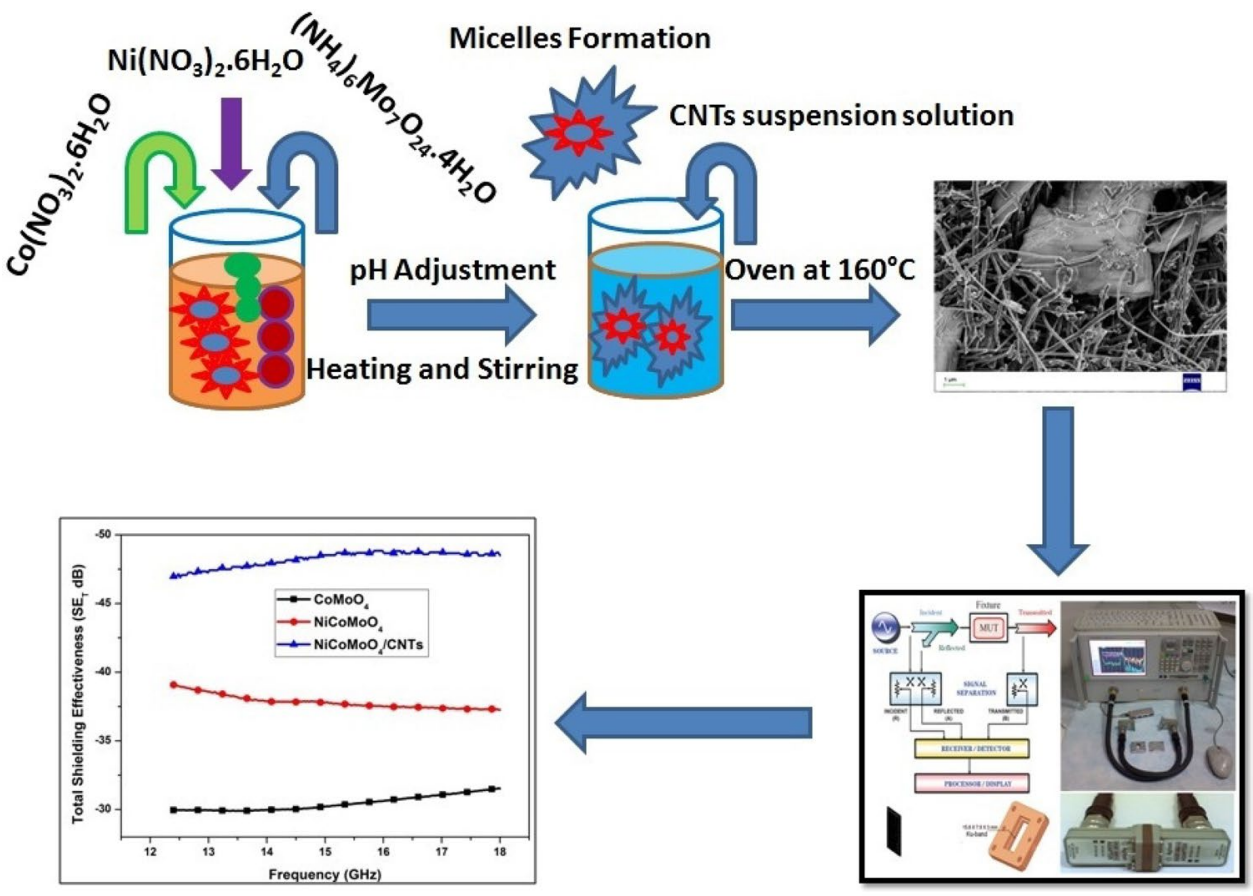

SN Applied Sciences A SPRINGER NATURE journal 

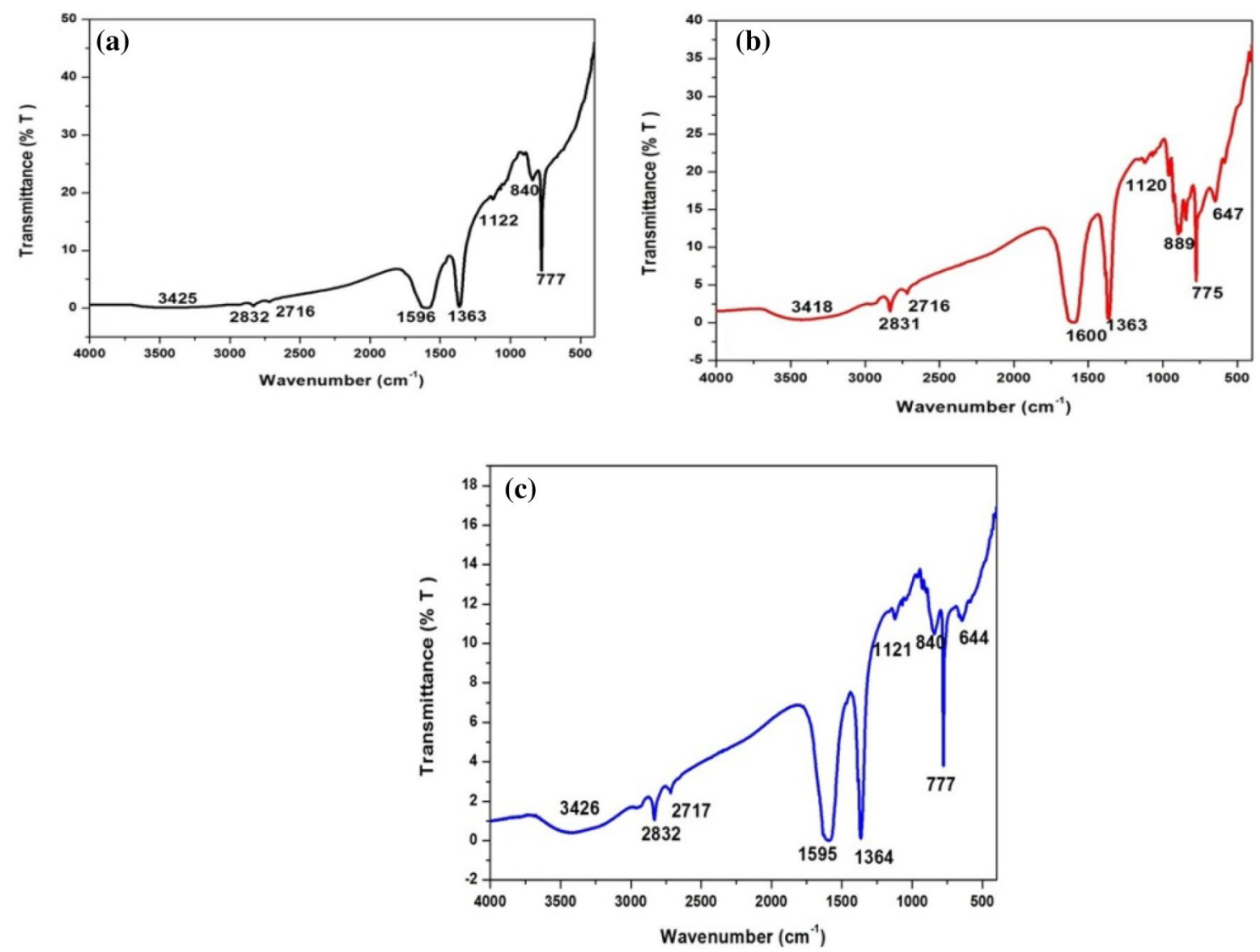

Fig. 2 FTIR spectra of a cobalt molybdate, b nickel cobalt molybdate and $\mathbf{c}$ nickel cobalt molybdate/CNT composite

840 and $889 \mathrm{~cm}^{-1}$ which are characteristic bands of tetrahedral $\mathrm{MoO}_{4}$ groups $[4,5]$ were observed, and their presence authenticates the formation of tetrahedral coordination of Mo on the surface of $\mathrm{a}-\mathrm{MoO}_{4}$.

\subsection{Structural studies of synthesized samples}

The crystalline structures of the as-synthesized $\mathrm{CoMoO}_{4}$ $\mathrm{NiCoMoO}$ and $\mathrm{NiCoMoO}_{4} / \mathrm{CNT}$ nanorods were confirmed by their XRD patterns, as shown in Fig. 3. The diffraction peaks at $2 \theta=23.2,27.1,29.3$ and 32.8 could be indexed as $\mathrm{CoMoO}_{4}$ (JCPDS Card No. 26-0477). The diffraction peaks at $2 \theta=29.4$ and 33.2 could be an indicator of Ni incorporation in $\mathrm{CoMoO}_{4}$ (JCPDS Card No. 13-0128). The diffraction peak at $2 \theta=27.2$ is weakened and the diffraction peak at $2 \theta=33.2$ gradually enhanced due to the incorporation of fourth component, i.e. CNTs. The sharp peak at $2 \theta=26.3$ revealed the presence of CNTs in $\mathrm{NiCOMoO}_{4} / \mathrm{CNT}$ composite $[4,5]$. The peaks corresponding to nickel and cobalt molybdate are also observed in $\mathrm{NiCoMoO}_{4} / \mathrm{CNT}$ composite and diffraction pattern indexed to (220), (311), (400) and (440) diffractions. Furthermore, the presence of sharp peak at $2 \theta=26.3$ and 43.4 revealed the presence of CNTs in the composite matrix. It is evident from X-ray diffraction pattern that $\mathrm{NiCoMoO}_{4} / \mathrm{CNT}$ composite has diffraction peaks of CNTs as well as nickel, cobalt and molybdate, thus confirming the incorporation of CNTs into nickel cobalt molybdate matrix.

\subsection{Morphological and EDS studies of $\mathrm{CoMoO}_{4}$, $\mathrm{NiCOMoO}_{4}$ and $\mathrm{NiCoMoO}_{4} / \mathrm{CNTs}$}

The size and morphology of as-synthesized $\mathrm{CoMoO}_{4}$, $\mathrm{NiCoMoO}$ and $\mathrm{NiCoMoO}_{4} / \mathrm{CNTs}$ were examined by field emission scanning electron microscopy (FE-SEM). Figure 4 shows typical FE-SEM images of as-synthesized $\mathrm{CoMoO}_{4}$, $\mathrm{NiCoMoO}$ and $\mathrm{NiCoMoO}_{4} / \mathrm{CNTs}$. It indicates that all the samples almost completely consist of nanorod structures [4-8]. The particles are shaped into irregular quasi rectangular nanorod with increasing size. The particles' size obtained for $\mathrm{CoMoO}_{4}, \mathrm{NiCoMoO}$ and $\mathrm{NiCoMoO} /$ CNTs from SEM morphology is in the range of 45-65 nm, $75-85 \mathrm{~nm}$ and $100-125 \mathrm{~nm}$. It is observed that after $\mathrm{Ni}$ doping in $\mathrm{CoMoO}_{4}$ particle size is enlarged and particles are transformed into uniform size with better morphology. The SEM image of $\mathrm{NiCOMoO}_{4} /$ CNTs shows the thread-like 

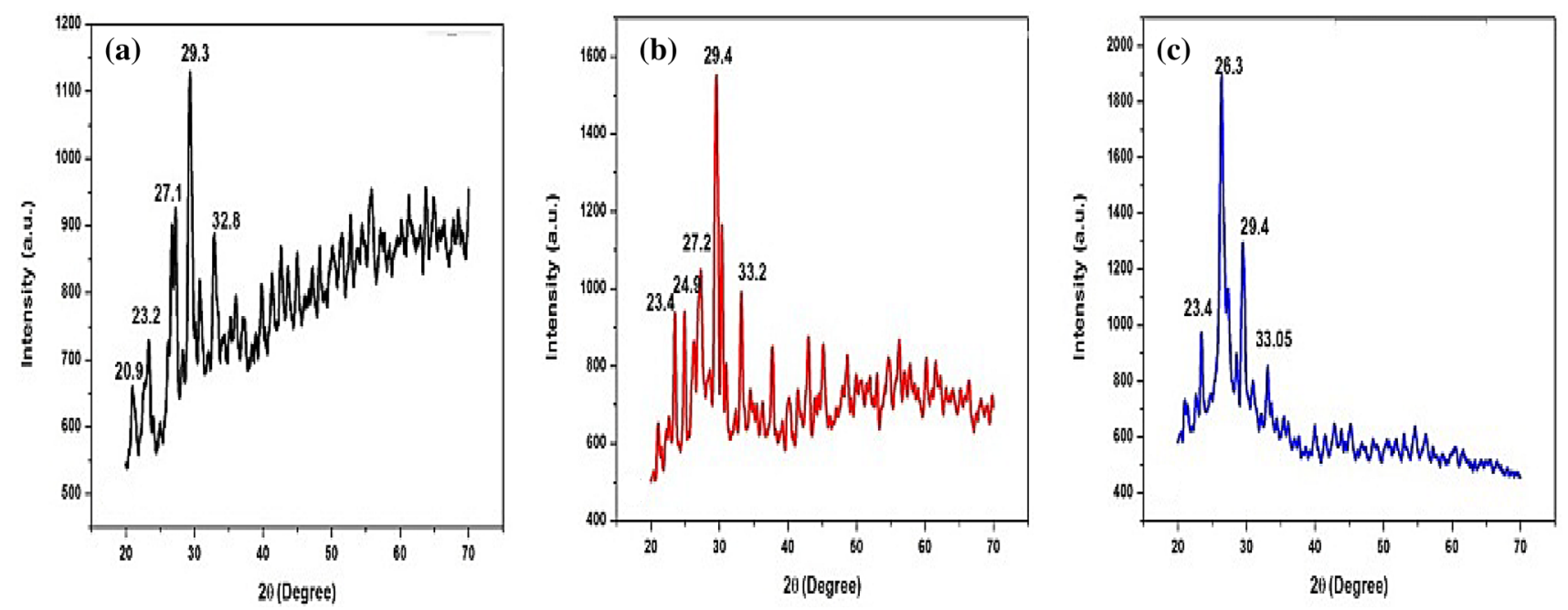

Fig. 3 XRD patterns of $\mathbf{a} \mathrm{CoMoO}_{4}, \mathbf{b} \mathrm{NiCoMoO}_{4}$ and $\mathbf{c} \mathrm{NiCoMoO}_{4} / \mathrm{CNT}$ composite
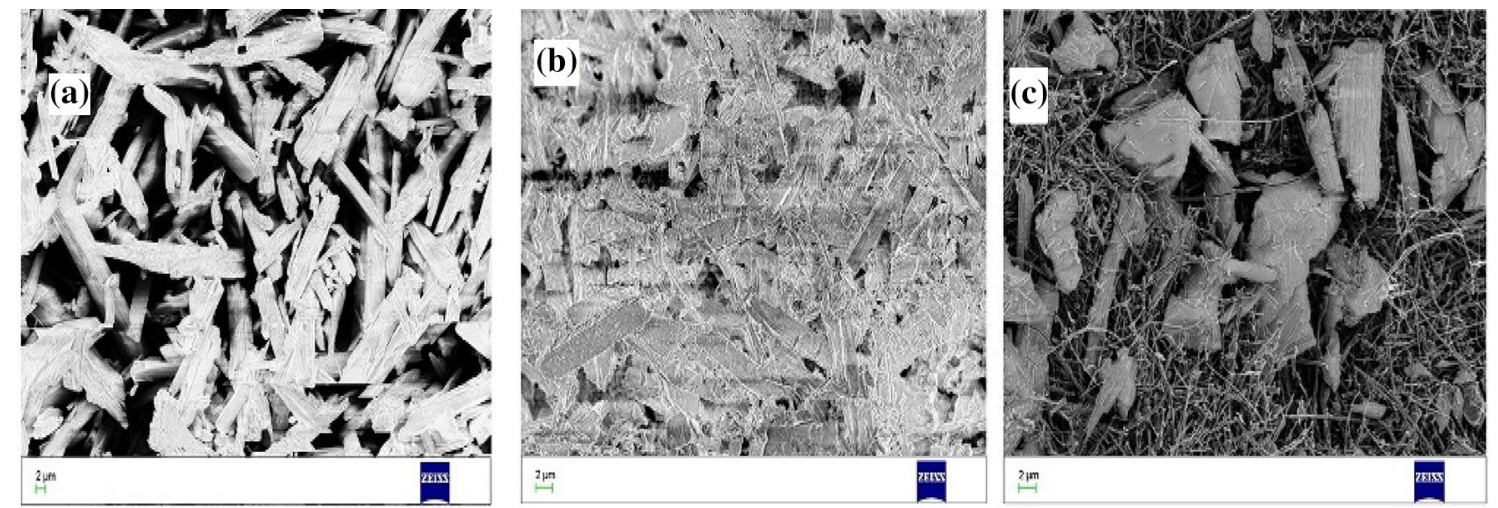

Fig. 4 FE-SEM images of $\mathbf{a} \mathrm{CoMoO}_{4}, \mathbf{b} \mathrm{NiCoMoO}_{4}$ and $\mathbf{c}$ NiCoMoO$/$ /CNT composite EDS analysis were taken to recognize the elemental composition of $\mathrm{NiCOMOO}_{4} / \mathrm{CNT}$ sample

structures that reveal the presence of carbon nanotubes (CNTs) in the composite. Basically CNTs are inert in nature and they cannot easily interact or make bonding with other materials. In the present study, firstly we have done acid treatment so that functionalization of CNTs makes them intractable to the nickel cobalt molybdate matrix; from FE-SEM figure, it is predictable that CNTs are embedded in between metal-metal matrix and act as filler phase.

The corresponding EDS mappings of $\mathrm{NiCOMoO}_{4} / \mathrm{CNT}$ nanorods are shown in Fig. 5 to further confirm the ratio between $\mathrm{Co}, \mathrm{Ni}$ and $\mathrm{Mo}$ elements. It can be seen that there are four colour mapping images, which are sky blue- $-\mathrm{Ni}-\mathrm{K}$, violet-Mo-L, pink-C-K and purple-Co-K, respectively. The synthesized $\mathrm{NiCoMoO}_{4} / \mathrm{CNTs}$ showed a composition of C (K) $90.60 \%, N(K) 6.63 \%, \mathrm{Mo}(\mathrm{L}) 1.70 \%, \mathrm{Ni}$ (K) $0.60 \%$ and $\mathrm{Co}$ (K) $0.48 \%$. The percentage of each component in $\mathrm{NiCoMoO}_{4} /$ CNT sample indicates that the ratio of $\mathrm{Mo}$ to $\mathrm{Ni}$ and $\mathrm{Co}$ is close to what was calculated for this synthesis process (Table 1).

\subsection{Electromagnetic interference shielding effectiveness measurements}

Shielding effectiveness (SE) is generally used to enumerate the combined loss of EM radiations, which can be mathematically formulated as [19-23]

$\mathrm{SE}_{\text {tot }}=\mathrm{SE}_{\mathrm{R}}+\mathrm{SE}_{\mathrm{A}}+\mathrm{SE}_{\mathrm{M}}$.

$S E_{R}$ loss is the consequence of impedance deviation between free space and shielding materials. In an intense situation, zero reflection can be attained at the shielding material surface when the impedance is equal to the value of $377 \Omega \mathrm{sq}^{-1}$ (standard impedance of the free space). $\mathrm{SE}_{\mathrm{A}}$ loss is generally considered as the collective effect from 
Fig. 5 EDS spectrum of $\mathrm{NiCoMoO} /$ /CNTs

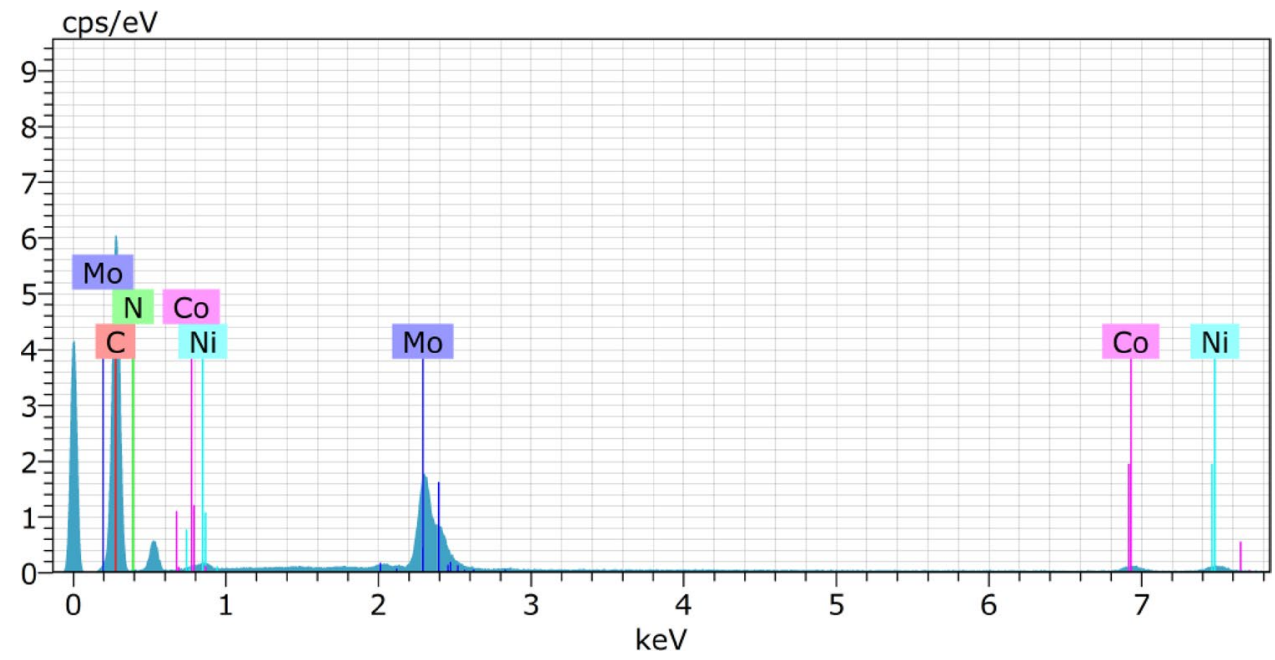

Table 1 Quantitative analysis of ratio between $\mathrm{Co}, \mathrm{Ni}$ and $\mathrm{Mo}$ elements was also tested as follows

\begin{tabular}{lclrrrr}
\hline El & AN & Series & {$[w t \%]$} & {$[w t \%]$} & [at.\%] & [wt\%] \\
\hline $\mathrm{C}$ & 6 & K series & 88.12 & 77.34 & 90.60 & 10.12 \\
$\mathrm{~N}$ & 7 & K series & 7.52 & 6.60 & 6.63 & 1.90 \\
$\mathrm{Mo}$ & 42 & L series & 13.18 & 11.57 & 1.70 & 0.50 \\
$\mathrm{Ni}$ & 28 & K series & 2.84 & 2.49 & 0.60 & 0.12 \\
$\mathrm{Co}$ & 27 & K series & 2.29 & 2.01 & 0.48 & 0.10 \\
\hline
\end{tabular}

Total: $113.95,100.00,100.00$

dielectric loss and magnetic loss. It is significantly revealed that the match level between magnetic loss and dielectric loss is a determinative factor in this loss mode. Mere high dielectric loss or high magnetic loss will surely give increase in lacking $\mathrm{SE}_{\mathrm{A}}$ loss on account of the inequity of the EM match. $\mathrm{SE}_{\mathrm{M}}$ loss is recognized as the spreading effect of the inhomogeneous material, which can be ignored due to the minute involvement in the collective loss when $\mathrm{SE}_{\text {tot }} \geq 15$ decibels $(\mathrm{dB})$. Therefore, SE for highperformance EMI shielding can be approximately simplified as $\mathrm{SE}_{\text {tot }} \approx \mathrm{SE}_{\mathrm{R}}+\mathrm{SE}_{\mathrm{A}}$.

Additionally, the comparative intensity of the efficiently incident $E M$ wave within the materials after first reflection is based on the quantity $(1-\mathrm{R})$. The effective absorbance $\left(A_{\text {eff }}\right)$ can be described as $A_{\text {eff }}=(1-R-T) /(1-R)$ after normalization with respect to the power of the effectively incident $E M$ wave inside the shielding material. Therefore, it is convenient to express the reflection and effective absorption losses in the form of $-10 \log (1-R)$ and $-10 \log \left(1-A_{\text {eff }}\right)$, respectively [21-28], i.e. express $\mathrm{SE}_{\mathrm{R}}$ and $\mathrm{SE}_{\mathrm{A}}$ as follows

$\mathrm{SE}_{\mathrm{R}}=10 \log (1-R)$ and $\mathrm{SE}_{A}=10 \log \left(1-A_{\text {eff }}\right)=10 \log \frac{T}{1-R}$

The term skin depth $(\delta)$ is the depth of penetration at which the intensity of the electromagnetic wave is condensed to $1 / e$ of its original strength. The $\delta$ relies upon various parameters like angular frequency $(\omega)$, real relative permeability $\left(\mu^{\prime}\right)$ and total conductivity $\left(\sigma_{T}\right)$ and can be expressed as $\delta=\sqrt{2 / \omega \mu \sigma_{\mathrm{T}}}$. Therefore, at any given frequency, skin depth decreases with the increase in both magnetic permeability and electrical conductivity. Further, for an electrically thick shield $(t>\delta)$, frequency $(\omega)$ dependence of far-field reflection and absorption losses can be expressed in terms of total conductivity $\left(\sigma_{T}\right)$ real permeability $\left(\mu^{\prime}\right)$, skin depth $(\delta)$ and thickness $(t)$ of the shield material as:

$\mathrm{SE}_{\mathrm{R}}(\mathrm{dB})=-10 \log \left(\frac{\sigma \mathrm{T}}{16 \omega \varepsilon_{o} \mu^{\prime}}\right)$

$\mathrm{SE}_{\mathrm{A}}(\mathrm{dB})=-20 \frac{t}{\delta} \log e=-8.68\left(\frac{t}{\delta}\right)$

This relationship attributes that SE value is directly proportional to thickness and conductivity and inversely proportional to the skin depth parameters. The $\sigma_{\mathrm{T}}$ and $\delta$ can be correlated with imaginary permittivity $\left(\varepsilon^{\prime \prime}\right)$ and real permeability $\left(\mu^{\prime}\right)$ as $\sigma_{\mathrm{T}}=\omega \varepsilon_{o} \varepsilon^{\prime \prime}$ and $\delta=\left(\frac{2}{\sigma T \omega \mu^{\prime}}\right)^{\frac{1}{2}}$. This gives absorption loss as $\mathrm{SE}_{\mathrm{A}}(\mathrm{dB})=-8.68 t\left(\frac{\sigma \mathrm{T} \omega \mu^{\prime}}{2}\right)^{\frac{1}{2}}$.

The thin skin depths and high conductivity $\left(\sigma_{\mathrm{T}}\right)$ values in the microwave region often result in the involvement of 

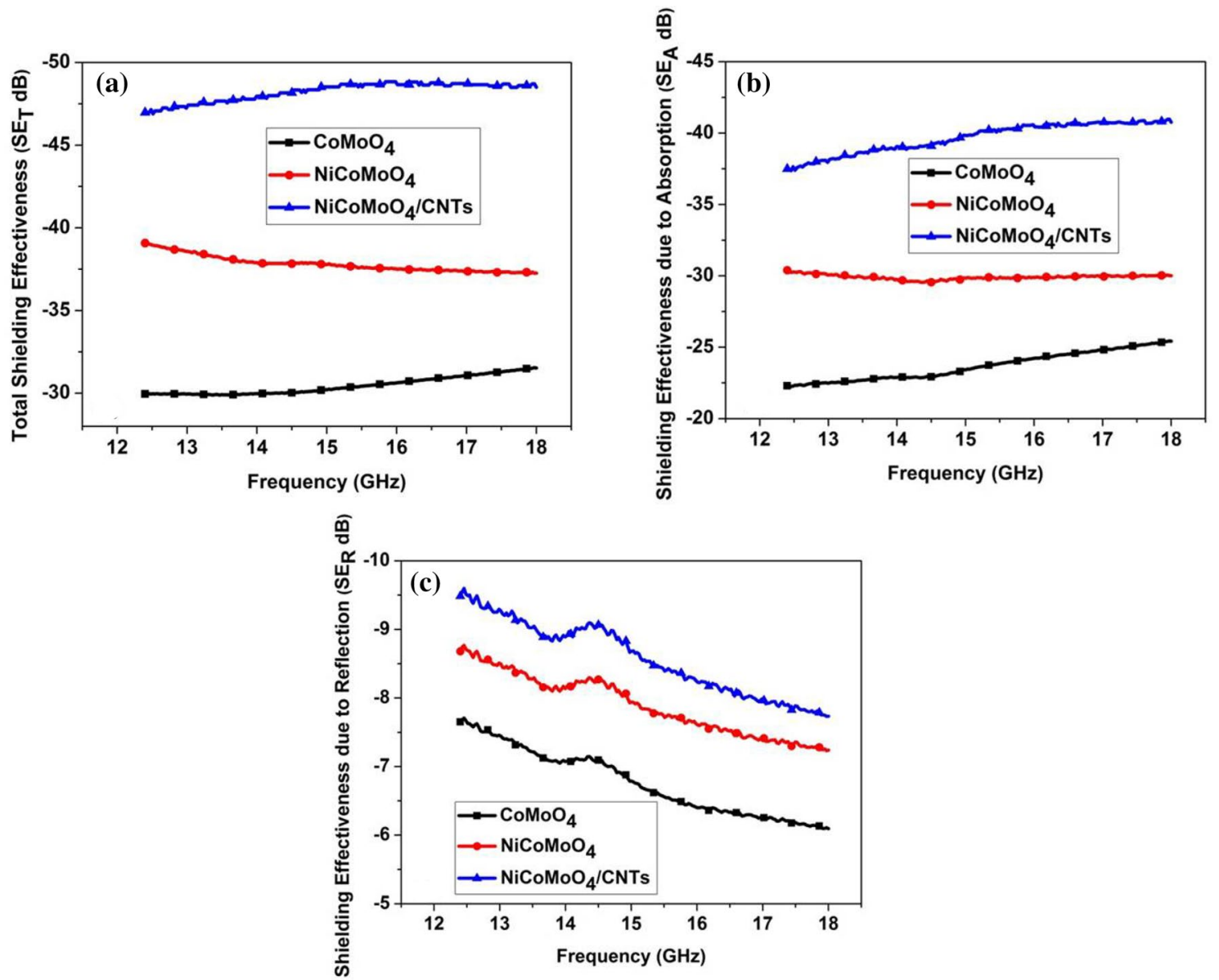

Fig. 6 a Variation in total shielding effectiveness with frequency of composites, b shielding effectiveness due to the absorption loss of composites $\mathbf{c}$ shielding effectiveness due to the reflection loss of composites

$\mathrm{SE}_{\mathrm{A}}$ becoming higher compared to $\mathrm{SE}_{\mathrm{R}}$. Therefore, absorption acts as leading shielding mechanism with apparent reflection values. Figure 6 (a) shows variations in total shielding effectiveness of $\mathrm{CoMoO}_{4}, \mathrm{NiCoMoO}_{4}, \mathrm{NiCoMoO}_{4} /$ CNT composites with frequency, (b) shows shielding effectiveness due to the absorption loss of composites and (c) shows shielding effectiveness due to the reflection loss of composites. As shown in Fig. 6, the $\mathrm{NiCoMoO}_{4} / \mathrm{CNTS}$ had higher EMI SE than $\mathrm{CoMoO}_{4}$ and $\mathrm{NiCoMoO}_{4}$. Table 2 summarizes the EMI shielding data of metal-metal molybdate/CNT composites. The total experimental SE was in the range of 31.54-48.84 dB within the Ku band frequency, representing a capability of $>99.9 \% \mathrm{EM}$ wave attenuation. The resultant EMI shielding materials could be classified as attenuation levels of "AAAAA" for common public use in consumptive electronic commodities and communication-related products. Furthermore, we discovered a significant EMI SE enhancement phenomenon in multilayered ferromagnetic $\mathrm{NiCoMoO}_{4}$-incorporated MWCNT composite. As shown in Fig. 6, the improved EMI
Table 2 EMI shielding data of metal-metal molybdate/CNT composites

\begin{tabular}{lllll}
\hline S. no. & Sample & $\mathrm{SE}_{\mathrm{T}}(\mathrm{dB})$ & $\mathrm{SE}_{\mathrm{A}}(\mathrm{dB})$ & $\mathrm{SE}_{\mathrm{R}}(\mathrm{dB})$ \\
\hline 1. & $\mathrm{CoMoO}_{4}$ & 31.54 & 25.43 & 6.10 \\
2. & $\mathrm{NiCoMoO}_{4}$ & 39.04 & 30.30 & 8.74 \\
3. & $\mathrm{NiCoMoO}_{4} / \mathrm{CNTs}$ & 48.84 & 40.56 & 8.27 \\
\hline
\end{tabular}

SE showed a frequency-dependent alteration throughout the Ku band region.

Doping of $\mathrm{Ni}$ in $\mathrm{CoMoO}_{4}$ nanorods and embedded into MWCNTs therefore improved the conductive pathway in the interior; this afford a high-efficiency conductive network for renovated EM energy into leaking current or heat. The dopants improved the conductivity, which in turn led to enhanced absorption of the EM radiation. The presence of coulomb interaction between the positively charged MWCNTs and negatively charged metallic nanorods enhanced the interfacial bonding. This improves the ability of dispersion of MWCNTs on the surface of $\mathrm{NiCoMoO}_{4}$ 
and strengthens their constancy. $\mathrm{NiCoMoO}_{4}$ nanorods help MWCNTs in preventing them from self-agglomeration and keeping them independent. Moreover, the high aspect ratio of MWCNTs provides large surface areas, which helped to form more active sites; all of these benefits resulted in a compact structure (as validated by XRD analysis); a compact metallic coating tends to have high EMI SE due to its high electric conductivity. As illustrated in Fig. 6, interphase and inner MWCNT incorporation played a very important role in the attenuation of EMI radiations to shield most of the incident energy. If we could take benefit of this EMI SE enhancement effect to the full extent by controlling the metal content, high-performance EMI shielding material with ultralightweight would certainly be obtained. Apparently, conductivity is an orthotropic influence factor in the $\mathrm{SE}_{\mathrm{A}}$, and the increase in $\mathrm{SE}$ results was in agreement with the relation given in the above equations. As a result, the $\mathrm{SE}_{\text {tot }}$ was found to considerably enhance with the loading of MWCNTs into $\mathrm{NiCoMoO}$ matrix and improved $\mathrm{SE}_{\text {tot }}$ was also predominantly due to the contribution from $\mathrm{SE}_{\mathrm{A}}$. We have investigated the alterations of $\mathrm{SE}_{\mathrm{R}}$ and $\mathrm{SE}_{\mathrm{A}}$ due to the introduction of MWCNTs into metal molybdate matrix. According to Fig. $6 b, c$, the loading of MWCNT coating onto metal molybdate matrix offered evident enhancements in both $\mathrm{SE}_{\mathrm{R}}$ and $\mathrm{SE}_{\mathrm{A}^{\prime}}$, showing up to $80 \%$ increment, respectively. $S E_{R}$ is dependent on the impedance conditions at the interface between shielding materials and free space. After loading of MWCNTs within Co-Ni-Mo multilayer ternary metal matrix, the surface bared to free space changed from $\mathrm{NiCoMoO}_{4}$ free space interface to CNT free space interface. Towards our information, $\mathrm{SE}_{\mathrm{A}}$ is the combined consequence of dielectric loss and magnetic loss and mainly determined by their match level. High dielectric/magnetic loss match level is beneficial to $\mathrm{EMI} \mathrm{SE}_{\mathrm{A}}$ enhancement. Generally, flexibility and lightweight account for a considerable proportion in designing and evaluating the shielding materials. Hence, these findings may provide guidelines for preparing highefficiency EMI shielding fabrics that have ultralightweight. Prospective investigation will be directed to examine the universality of this EMI SE enhancement phenomenon in other layered ferromagnetic metal/MWCNT blends.

The EM parameters, i.e. relative complex permittivity $\left(\varepsilon^{*}=\varepsilon^{\prime}-\mathrm{i} \varepsilon^{\prime \prime}\right)$ and relative complex permeability $\left(\mu^{*}=\mu^{\prime}-\mathrm{i} \mu^{\prime \prime}\right)$, have been calculated at room temperature for the study of electromagnetic wave absorption properties of composites which are shown in Fig. 7a-d and Table 3. These acquired complex parameters have been approximated from experimental scattering parameters (S11 and S21) by standard Nicholson, Ross and Weir theoretical calculations. The estimated real part of the EM parameters $\left(\varepsilon^{\prime}=25-50, \mu^{\prime}=0.45-0.68\right)$ is openly connected with the quantity of polarization occurring in the material which stands for the storage capability of the electric and magnetic energy, while the imaginary part $\left(\varepsilon^{\prime \prime}=70-138.69\right.$, $\left.\mu^{\prime \prime}=0.3-0.55\right)$ is imply for the self-indulgent electric and magnetic energy. From Fig. 7, for $\mathrm{NiCoMoO}_{4} / \mathrm{CNT}$ composite the values of $\varepsilon^{\prime}, \varepsilon^{\prime \prime}, \mu^{\prime}$ and $\mu^{\prime \prime}$ are in the range of $49.3,138.69,0.68$ and 0.55 , respectively. It is anticipated that MWCNTs may enhance the conductivity and electric polarization because the relative complex permittivity is a measure of the polarizability of a material which induces dipolar and electric polarization in the presence of microwave. Dielectric tangent loss $\left(\tan \delta \varepsilon=\varepsilon^{\prime \prime} / \varepsilon^{\prime}\right)$ and the magnetic tangent loss $\left(\tan \delta_{\mathrm{M}}=\mu^{\prime \prime} / \mu^{\prime}\right)$ of $\mathrm{CoMoO}_{4}, \mathrm{NiCoMoO}_{4}$, $\mathrm{NiCoMoO} / \mathrm{CNT}$ composites are also calculated using the permittivity and permeability parameters of the samples and represented in Fig. 8a, b, respectively. The experimental calculated values of $\tan \delta \varepsilon$ are $4-8$ and $\tan \delta_{M}$ are $0.6-1.2$ in the entire frequency range, indicating that the dielectric loss happened in all frequency ranges. The experiential dielectric tangent loss $(\tan \delta \varepsilon$ ) achieves up to 7.5 at $15 \mathrm{GHz}$ and continues higher than 7 over the whole region (Fig. 7), much higher than the magnetic loss, which designates that dielectric loss creates main involvement in the electromagnetic loss. These results also suggest that $\mathrm{NiCoMoO} /{ }_{4} / \mathrm{CNT}$ composite have distinct dielectric loss properties. According to the EM theory, dielectric losses are the effect of multifaceted phenomenon like ordinary resonance, dipole recreation, electronic polarization and its relaxation in the metal molybdate matrix and their relaxation and certainly the unique structure of the shield. $\mathrm{NiCoMoO}$ nanorod-tinted MWCNTs, due to its elevated conductivity, act as a polarized centre inside the CNTs which results in more electromagnetic wave absorption. High aspect ratio of the MWCNTs filled with $\mathrm{NiCoMoO}_{4}$ nanorods having high conductivity also enhances the absorption properties. Ferromagnetic nanorods act as minute dipoles which acquire polarization in the existence of EM field and result in electromagnetic wave absorption.

Interfacial polarization between MWCNT and $\mathrm{NiCOMoO}_{4}$ nanorods is responsible for further dielectric losses. Interfacial polarization occurs in heterogeneous media due to the accumulation of charges at the interfaces and the formation of large dipoles. The magnetic loss $\left(\tan \delta_{\mathrm{M}}\right)$ is an outcome of eddy current effects, natural resonances and anisotropy energy present in the composites. In the microwave ranges, the presence of ferromagnetic nanorods in the composite is the main cause of eddy current. However, the higher value of anisotropy energy also increases $\mathrm{SE}$ due to the microwave absorption. $\mathrm{SE}_{\mathrm{A}}$ becomes more dominating in contrast to the $S E_{R}$ in the microwave range. This may be caused by the high conductivity $\left(\sigma_{\mathrm{s}}=53\right.$ to $101 \mathrm{~S} / \mathrm{m}$, Fig. 8c) and shallow skin depth (Fig. 8d $\delta=0.5$ to $1.10 \mu \mathrm{m}$ ) values at such high frequencies. From the experimental measurement, the SE due to the absorption 

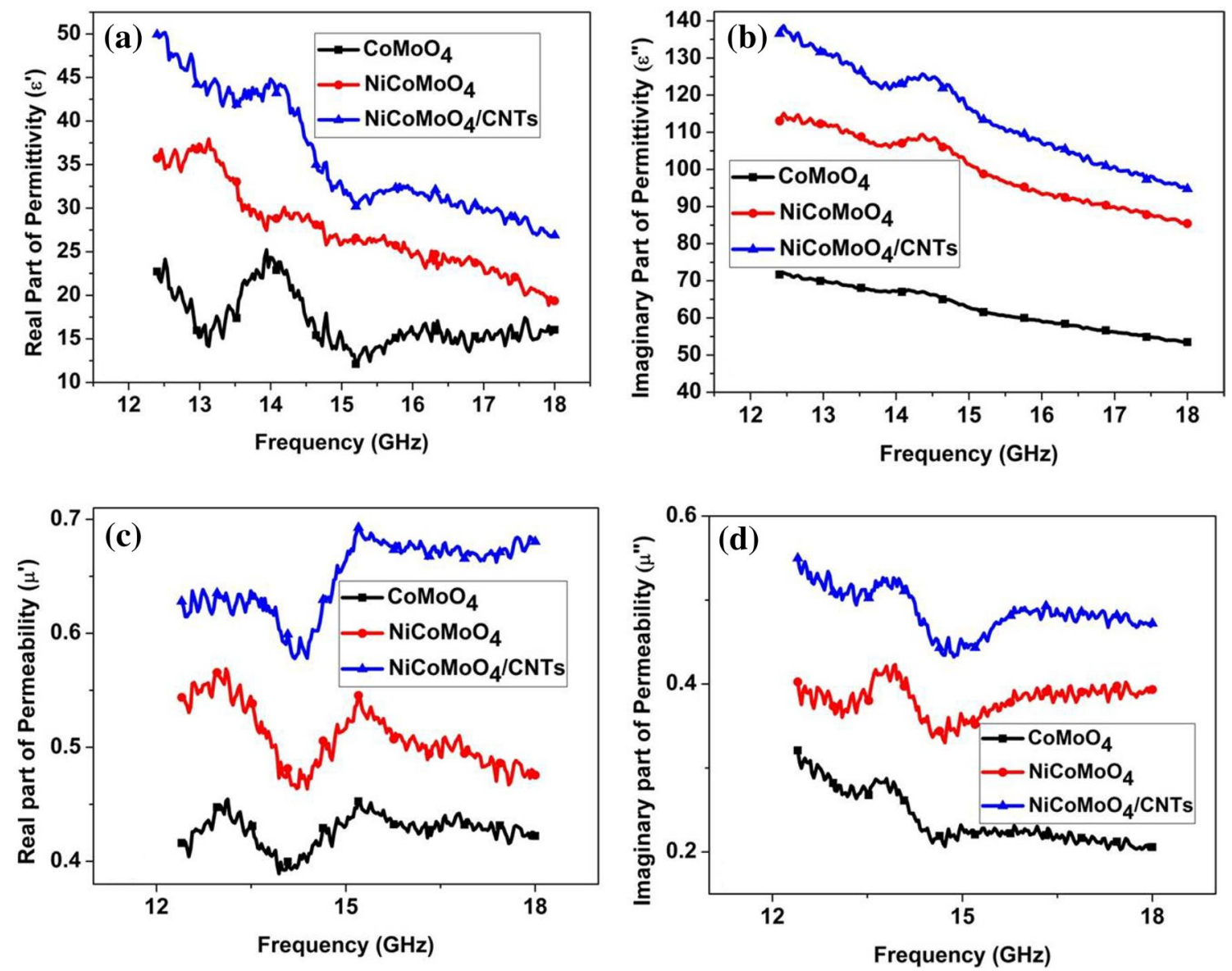

Fig. 7 Variations in $\mathbf{a} \varepsilon^{\prime}, \mathbf{b} \varepsilon^{\prime \prime}, \mathbf{c} \mu^{\prime}$ and $\mathbf{d} \mu^{\prime \prime}$ with frequency, the real and imaginary parts of the complex permittivity $\varepsilon_{\mathrm{r}}$ and complex permeability $\mu_{\mathrm{r}}$ of composites

Table 3 Permittivity, permeability, dielectric tangent loss, magnetic tangent loss, specific conductivity $\left(\sigma_{s}\right)$ and skin depth $(\delta)$ of metal-metal molybdate/CNT composites

\begin{tabular}{llllllllll}
\hline S. no. & Sample & $\varepsilon^{\prime}$ & $\varepsilon^{\prime \prime}$ & $\mu^{\prime}$ & $\mu^{\prime \prime}$ & $\tan \delta \varepsilon=\varepsilon^{\prime \prime} / \varepsilon^{\prime}$ & $\tan \delta_{\mathrm{M}}=\mu^{\prime \prime} / \mu^{\prime}$ & $\sigma_{\mathrm{s}}(\mathrm{S} / \mathrm{m})$ & $\delta(\mu \mathrm{m})$ \\
\hline 1. & $\mathrm{CoMoO}_{4}$ & 25 & 70 & 0.4 & 0.3 & 2.8 & 0.65 & 53 \\
2. & $\mathrm{NiCoMoO}_{4}$ & 37 & 110 & 0.55 & 0.4 & 3 & 0.72 & 1.10 \\
3. & $\mathrm{NiCoMoO}_{4} / \mathrm{CNTS}$ & 50 & 140 & 0.69 & 0.55 & 2.85 & 1.25 & 0.7 \\
\hline
\end{tabular}

$\left(\mathrm{SE}_{\mathrm{A}}\right)$ of composites has been found to vary from 25 to $40 \mathrm{~dB}$, while the $S E_{R}$ remains nearly constant at $8 \mathrm{~dB}$. Thus, the total SE achieved for the composite is dominated by absorption, while the $\mathrm{SE}_{\mathrm{R}}$ is constant. The skin depth of $\mathrm{NiCoMoO} / 4$ CNT composite is very diminutive $(0.5 \mu \mathrm{m})$, due to the high electrical conductivity and high-quality magnetic properties. From equations, it is seen that better $\mathrm{SE}_{\mathrm{A}}$ can be achieved from moderate conducting materials. The outstanding EM waves absorbing performance of $\mathrm{NiCoMoO} / \mathrm{CNT}$ composite is mainly attributed to two factors: impedance matching and EM wave attenuation. The ideal condition for the perfect absorber is $\varepsilon_{\mathrm{r}}=\mu_{r}$ and the occurrence of MWCNTs decorated with ferromagnetic $\mathrm{NiCoMoO}{ }_{4}$ nanorods in the core of metal molybdate matrix has lowered $\varepsilon_{\mathrm{r}}$ of the composite and improved the equality of $\varepsilon_{r}$ and $\mu_{r}$, which helps to improve the level of impedance matching. Also, $\mathrm{NiCoMoO}_{4}$ nanorod composite have strong microwave absorption due to their high dielectric and magnetic loss. However, from our experimental observations with MWCNTs and many other conductive types of filler such as carbon black, we can definitely claim that conductivity, and therefore connectivity, is required in order to produce a better composite for EMI shielding. Therefore, more research is required to comprehend the relationship 

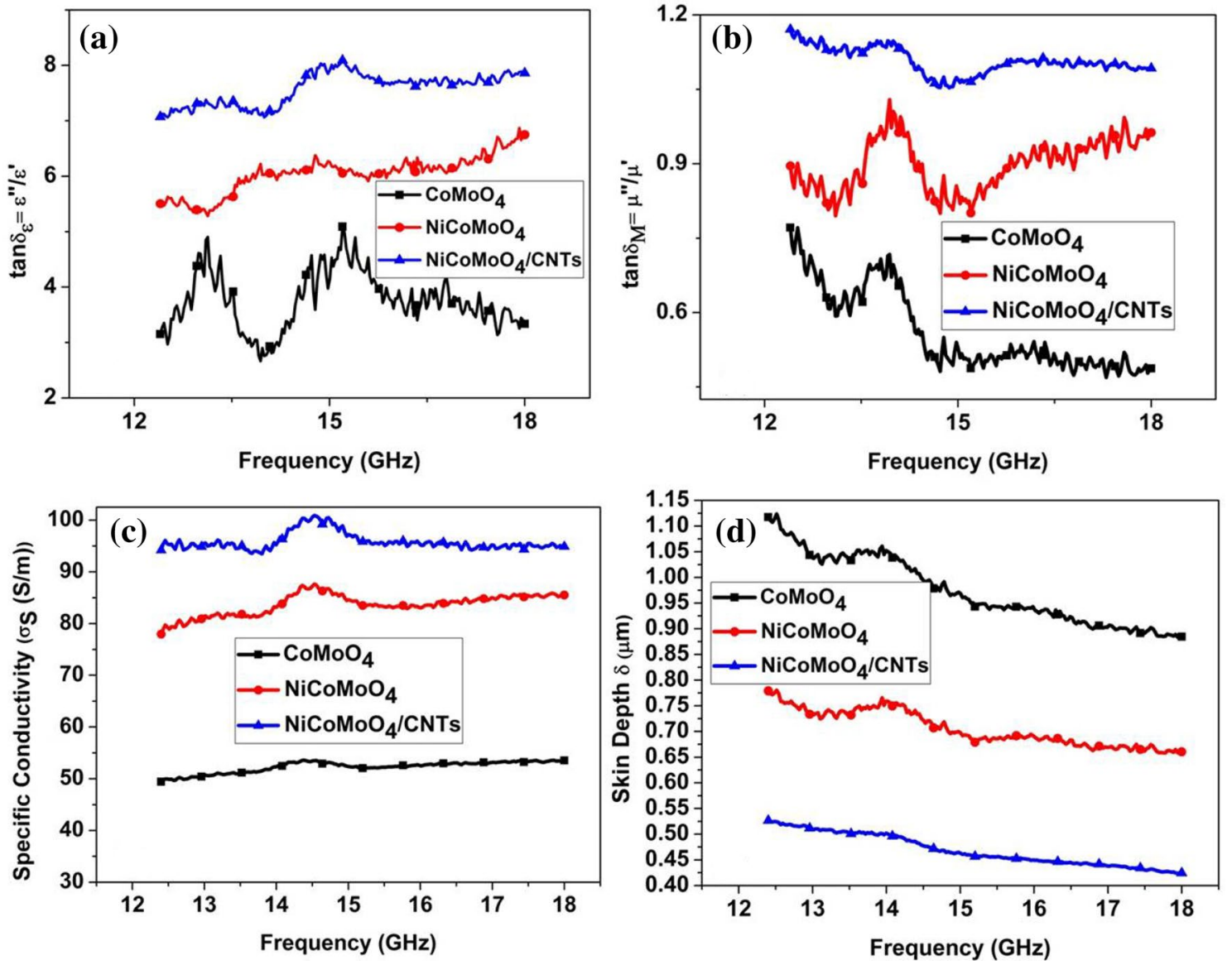

Fig. 8 Variations in a loss tangent of the dielectric $\tan \delta_{\mathrm{E}}=\varepsilon^{\prime \prime} / \varepsilon^{\prime}$ with frequency, $\mathbf{b}$ variations of loss tangent of the magnetic tan $\delta_{\mathrm{M}}=\mu^{\prime \prime} / \mu^{\prime}, \mathbf{c}$ specific conductivity $\mathrm{v} / \mathrm{s}$ frequency and $\mathbf{d}$ skin depth variations with frequency

between the conductivity and EMI SE of metal-metal molybdate/MWCNT composites. It is worth point out here that other factors such as filler dispersion, intrinsic conductivity, distribution and orientation might have significant influence on the EMI shielding of a metal molybdate/ MWCNT composite. For EMI shielding by absorption, we think that composite conductivity is an important factor in addition to the filler intrinsic conductivity and concentration. The evaluations here recommend that the microwave absorption with respect to both dielectric and magnetic losses demonstrates much more valuable performance. In this observation, the grouping of magnetic materials with dielectric materials has been demonstrated to afford a capable arena for attaining high-performance microwave absorption materials. Typically, multiphase heterostructures, such as $\mathrm{NiCOMoO}_{4} / \mathrm{CNT}$ composite, by introducing magnetic materials onto/into carbon nanotubes reveal enhanced resonance at the established interfaces and thus get better microwave absorption efficiency and interrelated effective bandwidth. On the other hand, electrical properties based on electrical conductivity could be play an important role in contrast to dielectric properties and microwave absorption. As already suggested by the results here, $\mathrm{NiCoMoO}_{4} / \mathrm{CNTs}$ is superior to $\mathrm{NiCoMoO}$, since CNTs are outstanding fillers for enhancing electromagnetic wave absorption based on both enhanced dielectric and magnetic properties. The preface of a second or multiple phases in/onto carbon materials through a precise approach plays a momentous role in design and manufacture of high-performance microwave absorption composites. Furthermore, for future aspects, we can improve the reflection/absorption coefficients and shielding effectiveness by introducing multiwalled carbon nanotubes with metal-metal matrix particles embedded in a phosphate ceramic matrix [29]. 


\section{Conclusion}

In summary, we have successfully synthesized cobalt molybdate, nickel cobalt molybdate and nickel cobalt molybdate/CNT composite nanorods through a facile hydrothermal method without the assistance of surfactants. FTIR, UV-Vis and EDX studies confirm the chemical composition of the synthesized samples. All samples show nanorod morphology. The particles' size obtained for $\mathrm{CoMoO}_{4}, \mathrm{NiCoMoO}_{4}$ and $\mathrm{NiCoMoO}_{4} / \mathrm{CNTs}$ from SEM morphology is in the range of $45-65 \mathrm{~nm}, 75-85 \mathrm{~nm}$ and 100-125 $\mathrm{nm}$. It has been concluded that after Ni doping in $\mathrm{CoMoO}_{4}$ particle size is increased and particles are changed into uniform size with better morphology. The SEM image of $\mathrm{NiCoMoO}_{4} / \mathrm{CNTs}$ shows the thread-like structures that reveal the presence of carbon nanotubes (CNTs) in the composite. EDS analysis was performed to identify the elemental composition of $\mathrm{NiCoMoO}_{4} / \mathrm{CNT}$ sample. The synthesized $\mathrm{NiCoMoO}_{4} / \mathrm{CNTs}$ showed a composition of C (K) $90.60 \%, N(K) 6.63 \%$, Mo (L) $1.70 \%, \mathrm{Ni}(\mathrm{K})$ $0.60 \%$ and $\mathrm{Co}(\mathrm{K}) \%$. The percentage of each component in $\mathrm{NiCoMoO}_{4} / \mathrm{CNT}$ sample specifies that the proportion of $\mathrm{Mo}$ to $\mathrm{Ni}$ and $\mathrm{Co}$ is close to what was calculated for this synthesis process. The core-shell nanorod tubular structure associated with MWCNTs embedded in metal-metal molybdate matrix responsible for the improved interfacial polarization and the responsible for improved anisotropy energy of composite; as a result, more and more dissemination occurs which lead to the high $\mathrm{SE}\left(\mathrm{SE}_{T} \sim 48.84 \mathrm{~dB}\right)$ in correlation with conventional materials. The synthesized novel nanorods have better EM wave absorption properties $\left(\mathrm{SE}_{\mathrm{A}} \sim 40 \mathrm{~dB}\right)$ which strongly depends upon optimal infilling of MWCNTs in metal molybdate matrix. Thus, the obtained results propose that the prepared composites are new potential class of EM wave absorption material.

\section{Compliance with ethical standards}

Conflict of interest The authors declare that they have no competing interests.

\section{References}

1. Ahlbom A, Cardis E, Green A, Linet M, Savitz D, Swerdlow A (2001) Review of the epidemiologic literature on EMF and health. Environ Health Perspect 109(supplement 6):911-933

2. World Health Organization (2007) Extremely low frequency fields, vol 238 of environmental health criteria monograph. WHO, Geneva

3. World Health Organization (2011) IARC classifies radiofrequency electromagnetic fields as possibly carcinogenic to humans. International Agency for Research Cancer, Lyon
4. Pang H, Lu QY, Zhang YZ, Li YC, Gao F (2010) Selective synthesis of nickel oxide nanowires and length effect on their electrochemical properties. Nanoscale 2:920

5. Pang H, Deng JW, Yan B, Ma YH, Li GC, Ai YN, Chen J, Zhang JS, Zheng HH, Du JM (2012) Cupric oxide nanorods on double-face copper micro puzzles electrode as promising anode materials for lithium ion batteries. Int J Electrochem Sci 7:10735

6. Pang H, Yan ZZ, Wang WQ, Wei YY, Li XX, Li J, Chen J, Zhang JS, Zheng $\mathrm{HH}$ (2012) Template-free controlled fabrication of $\mathrm{NH}_{4} \mathrm{MnPO}_{4} \cdot \mathrm{H}_{2} \mathrm{O}$ and $\mathrm{Mn}_{2} \mathrm{P}_{2} \mathrm{O}_{7}$ micro-nanostructures and study of their electrochemical properties. Int J Electrochem Sci 7:12340

7. Zhou KB, Wang X, Sun XM, Peng Q, Li YD (2005) Enhanced catalytic activity of ceria nanorods from well-defined reactive crystal planes. J Catal 229:206-212

8. Burda C, Chen X, Narayanan R, El-Sayed MA (2005) Chemistry and properties of nanocrystals of different shapes. Chem Rev 105:1025

9. Ghoshal T, Biswas S, Kar S (2008) Synthesis of Ag/Si core/shell coaxial nanowire heterostructures by the vapor-liquid-solid technique. J Phys Chem C 112:20138

10. Acharya S, Panda AB, Efrimaa S, Golan Y (2007) Polarization properties and switchable assembly of ultranarrow $\mathrm{ZnSe}$ nanorods. Adv Mater 19:1105

11. Acharya S, Patla I, Kost J, Efrima S, Golan Y (2006) Switchable assembly of ultra narrow CdS nanowires and nanorods. J Am Chem Soc 128:9294

12. Zhao DD, Xu MW, Zhou WJ, Zhang J, Li HL (2008) Preparation of ordered mesoporous nickel oxide film electrodes via lyotropic liquid crystal template electrodeposition route. Electrochim Acta 53:2699

13. Xiong SL, Yuan CZ, Zhang XG, Xi BJ, Qian YT (2009) Controllable synthesis of mesoporous $\mathrm{Co}_{3} \mathrm{O}_{4}$ nanostructures with tunable morphology for application in supercapacitors. Chem Eur J 15:5320

14. Hall PJ, Mirzaeian M, Fletcher SI, Sillars FB, Rennie AJR, ShittaBey GO, Wilson G, Cruden A, Carter R (2010) Energy storage in electrochemical capacitors: designing functional materials to improve performance. Energy Environ Sci 3:1238

15. Rodriguez J, Chaturvedi S, Hanson J, Albornoz A (1998) Electronic properties and phase transformations in $\mathrm{CoMoO}_{4}$ and $\mathrm{NiMoO}_{4}$ : XANES and time-resolved synchrotron XRD studies. J Phys Chem B 102:1347

16. Chu WG, Wang HF, Guo YJ, Zhang LN, Han ZH (2009) Catalyst-free growth of quasi-aligned nanorods of single crystal $\mathrm{Cu}_{3} \mathrm{Mo}_{2} \mathrm{O}_{9}$ and their catalytic properties. Inorg Chem 48:1243

17. Vilminot $S$, André G, Kurmoo M (2009) Magnetic properties and magnetic structure of $\mathrm{Cu}(\mathrm{II})_{3} \mathrm{Mo}(\mathrm{VI})_{2} \mathrm{O}_{9}$. Inorg Chem 48:2687

18. Ajayan PM, Stephan O, Colliex C (1994) Aligned carbon nanotube arrays formed by cutting a polymer resin-nanotube composite. Trauth Sci 265:1212

19. Xie XL, Mai YW, Zhou XP (2005) Dispersion and alignment of carbon nanotubes in polymer matrix: a review. Mater Sci Eng R49:89

20. Saini P (2009) Polyaniline-MWCNT nanocomposites for microwave absorption and EMI shielding materials. Chem Phys 113:919-926

21. Qiang R, Du YC, Zhao HT, Wang Y, Tian CH, Li ZG, Han XJ, Xu $P$ (2015) Metal organic framework-derived Fe/C nanocubes toward efficient microwave absorption. J Mater Chem A 3(25):13426-13434

22. Bhardwaj P, Kaushik S, Gairola P, Gairola SP Exceptional electromagnetic radiation shielding performance and dielectric properties of surfactant assisted polypyrrole-carbon allotropes 
composites. Radiat Phys Chem. https://doi.org/10.1016/j.radph yschem.2018.06.001

23. Sharma AK, Bhardwaj P, Singh KK, Dhawan SK Improved microwave shielding properties of polyaniline grown over threedimensional hybrid carbon assemblage substrate. Appl Nanosci. https://doi.org/10.1007/s13204-014-0362-x

24. Zhao H, Hou L, Bi S, Lu Y Enhanced X-band electromagnetic interference shielding performance of layer-structured fabricsupported polyaniline/cobalt-nickel coatings. ACS Appl Mater Interfaces. https://doi.org/10.1021/acsami.7b07941

25. Cao Mao-Sheng, Yang Jian, Song Wei-Li, Zhang De-Qing, Wen Bo (2012) Ferroferric oxide/multiwalled carbon nanotube vs polyaniline/ferroferric oxide/multiwalled carbon nanotube multiheterostructures for highly effective microwave absorption. ACS Appl Mater Interfaces 4:6949-6956

26. Bhattacharjee Yudhajit, Bhingardive Viraj, Biswas Sourav, Bose Suryasarathi (2016) Construction of a carbon fiber based layer-by-layer (LbL) assembly-a smart approach towards effective EMI shielding. RSC Adv 6:112614

27. Li N, Hu CG, Cao MH (2013) Enhanced microwave absorbing performance of CoNi alloy nanoparticles anchored on a spherical carbon monolith. Phys Chem Chem Phys 15(20):7685-7689

28. Jia LC, Yan DX, Cui CH, Jiang X, Ji X, Li ZM (2015) Electrically conductive and electromagnetic interference shielding of polyethylene composites with devisable carbon nanotube networks. J Mater Chem C 3(36):9369-9378

29. Bychanok Dzmitry, Gorokhov Gleb, Meisak Darya, Plyushch Artyom, Kuzhir Polina, Sokal Alexey, Lapko Konstantin, SanchezSanchez Angela, Fierro Vanessa, Celzard Alain, Gallagher Cameron, Hibbins AlastairP, Ogrin FeodorY, Brosseau Christian (2016) Exploring carbon nanotubes $/ \mathrm{BaTiO}_{3} / \mathrm{Fe}_{3} \mathrm{O}_{4}$ nanocomposites as microwave absorbers. Prog Electromagn Res C 66:77-85 